\title{
Quantifying Intra- and Intermolecular Phenomena: Challenging yet Exciting Territory for Quantum Chemistry
}

\author{
Clémence Corminboeuf ${ }^{\star}$
}

Werner Prize 2014

\begin{abstract}
Waals interactions occur in all molecules and intermolecular complexes but are poorly described by the most widely used electronic structure framework. This paper addresses the efforts pursued in our laboratory to improve the performance of standard density functional approximations and deliver modern strategies to analyze and fine-tune the underlying physics of intra- and intermolecular interactions.
\end{abstract}

Keywords: Density functional theory · Energy decomposition schemes · Non-covalent interactions

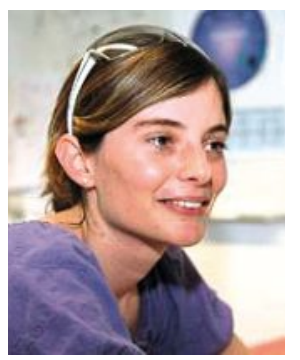

Clé me n ce Corminboeuf was appointed Tenure Track Assistant Professor and Sandoz Family Foundation Chair at the Ecole Polytechnique Fédéral de Lausanne in 2007. She earned her $\mathrm{PhD}$ from the University of Geneva, followed by postdoctoral stays at New York University and the University of Georgia (USA). Her research is essentially built upon the framework of Kohn-Sham density functional theory. Her group has been involved in the development and application of innovative methods for accurately describing and analyzing non-covalent interactions. She has co-authored more than 90 publications. In 2010, she was the silver medalist of the European Young Chemist Award. In 2012, she was awarded an ERC starting grant and received the Werner Prize of the Swiss chemical society in 2014.
${ }^{\star}$ Correspondence: Prof. Dr. C. Corminboeuf Laboratory for Computational Molecular Design Institut des Sciences et Ingénierie Chimiques Ecole Polytechnique Fédérale de Lausanne Avenue Forel 2

$\mathrm{CH}-1015$ Lausanne

Tel.: +41216939357

E-mail: clemence.corminboeuf@epfl.ch
Weak non-covalent interactions (i.e. dispersion) govern countless chemical phenomena ranging from complex supramolecular architectures to the condensed phases of non-polar organic molecules. They are also responsible for the intercalation of DNA exploited in chemotherapeutic treatments and for the regio-divergent pathways at the heart of asymmetric catalysis. On this basis, quantum chemistry represents a practical tool to determine how best to fully utilize these omnipresent interactions and elucidate their underlying physics. The research effort in our laboratory has been placed on extending the set of computational tools available through the development of improved and innovative approaches that accurately describe, characterize and interpret the nature of noncovalent interactions. ${ }^{[1]}$ Over the last few years, we have tackled some of the known deficiencies of standard density-functional approximations for treating challenging dispersion interactions present not only in van der Waals complexes but in all systems and molecules. ${ }^{[2-4]}$ Our contributions include the elaboration of atom pairwise energy corrections that account for dispersion between and within molecules. ${ }^{[5-9]}$ Along with improving the description of intra- and intermolecular interactions jointly, we also engaged in the development of original theoretical approaches for analyzing and describing phenomena underpinned by non-covalent interactions. ${ }^{[10-15]}$ This article highlights some of our main achievements along these directions, as illustrated in Scheme 1.

\section{Accurate Treatment of Non- covalent Interactions}

Our research is essentially built upon the framework of Kohn-Sham density

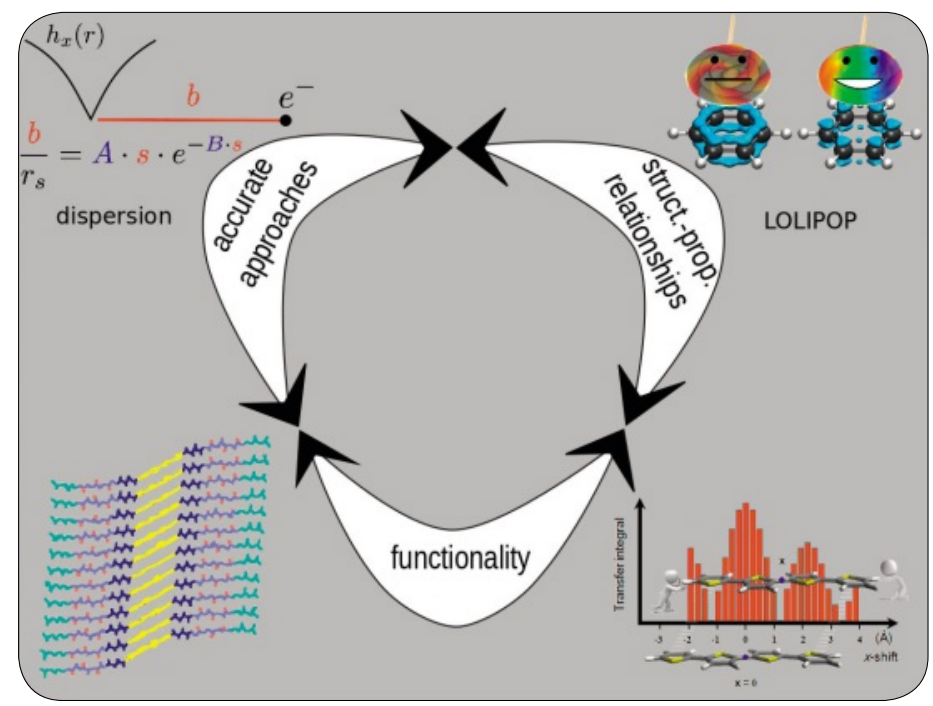

Scheme 1. 
functional theory (DFT), ${ }^{[16]}$ which offers a robust formalism and provides a practical balance of accuracy and computational cost for relatively large systems. In the context of chemistry, the breakthrough point occurred in mid-1990s with the introduction $^{[17]}$ and implementation, ${ }^{[18]}$ of the B3LYP functional. B3LYP not only provided accurate geometries but also good thermochemistry for a broad range of molecules and reaction pathways. Despite these advantages, the commonly used functional approximations (including B3LYP) intrinsically fail to recover the $\mathrm{R}^{-6}$ attractive form characteristic of dispersion interactions. ${ }^{[19-22]}$ The most obvious failures occur in situations where van der Waals interactions dominate the total binding energy, as exemplified by the repulsive interaction between neutral monomers at standard DFT levels. Note, however, that London dispersion interactions are ubiquitous; occurring between and within all molecules and governing key structural and electronic phenomena in chemistry, biology and material sciences. Over the last decade, the 'dispersion problem' stimulated a considerable amount of development in the density functional theory community following several distinct strategies. ${ }^{[23]}$ Achievements include the introduction of fully non-local van der Waals functionals (see e.g. refs [24] and [25]), the reintroduction of the dependence on virtual orbitals borrowed from wavefunction-based theory, ${ }^{[26-30]}$ the construction of dispersion-corrected atom-centered potentials, ${ }^{[31,32]}$ the fitting of flexible functional form (e.g. M06-2X[33]) and the atom-pairwise a posteriori energy corrections (see e.g. refs [34-40]). Atom pairwise dispersion corrections, perhaps, are the more commonly used approach to account for dispersion, but more recent schemes have also addressed the inclusion of many-body effects $^{[41]}$ and the importance of anisotropy. ${ }^{[42]}$

Atom pairwise dispersion corrections constitute a pragmatic and efficient solution that consists in adding the missing 1/ $\mathrm{R}^{6}$ energy correction terms a posteriori to a DFT computation:

$$
E_{d i s p}=-\sum_{i=1}^{N_{a t}} \sum_{i>j}^{N_{a t}} f_{d}\left(R_{i j}\right) \frac{C_{6}^{i j}}{R_{i g}^{6}}
$$

where $N_{\text {at }}$ is the number of atoms in the molecule or complex, $\mathrm{R}_{i j}$ is the internuclear distance between atom $i$ and $j$ and $\mathrm{C}_{6}{ }^{i j}$ is the associated dispersion coefficients. $f_{d}\left(R_{i j}\right)$ is a damping function that serves to attenuate or switch off the energy correction at shorter distances where the density functional approximation behaves properly. The pragmatic approach, originally proposed for Hartree-Fock ${ }^{[43]}$ prior to having an incidence on the DFT community, ${ }^{[44]}$ gained popularity through Grimme's work under the DFT-D acronym. ${ }^{[34-36]}$ Multiple flavors were subsequently developed, ranging from the classical schemes in which the $\mathrm{C}_{6}$ coefficients and the damping function parameter are tabulated a priori to systemdependent ${ }^{[37]}$ and even density-dependent corrections. ${ }^{[6-9,38-40]}$ Interested readers are referred to refs $[1,23,24]$ for more information on available approaches.

In contrast to other schemes, our primary concern was not to establish a correction for improving the description of intermolecular complexes but rather to create a variant that accounted for dispersion interactions present within molecules without any deterioration of the description of longer-range intermolecular interactions. ${ }^{[1,4-9]}$ Our strategy emphasized the introduction of more physics into both the damping function and the dispersion coefficients (i.e. the two characteristic ingredients of a dispersion correction), ultimately resulting with the incorporation of density-dependency in both ingredients ${ }^{[6,7]}$ as illustrated by our most established correction, $\mathrm{dDsC}{ }^{[8]}$ This approach was shown to improve the interaction energy of a variety of standard density functionals simultaneously for both typical intermolecular complexes and shorter-range interactions occurring within molecules.

We have recently reviewed our contributions to this domain ${ }^{[1]}$ and will refrain from discussing additional details and overall performance characteristics of $\mathrm{dDsC}$ here. Instead, we consider relevant examples that highlight the manifestation of intramolecular dispersion interactions and stress the importance of consistently using a dispersion correction. Fig. 1 nicely illustrates this by comparing the DFT $(-\mathrm{dDsC})$ reaction energies, geometries, and dynamic trajectories in the gas phase. The errors from high-level reference values of the non-corrected B3LYP reaction energies (A and $\mathbf{B}$ ) are striking, but agreement is restored if B3LYP-dDsC is used. In $\mathbf{C}$, underestimation of the $\pi$-stacking interaction leads to an overestimation of the inter-ring distances at the B3LYP level. ${ }^{[8]}$ D magnificently demonstrates how this overestimation accumulates in a BornOppenheimer molecular dynamic trajectory, ultimately resulting in a qualitatively incorrect evolution of the intermolecular distance over time. ${ }^{[45]}$ Obviously, the examples given in Fig. 1 are based on gas phase, ground state situations. They do not directly mirror those experimental conditions present in condensed phase or processes occurring in the excited state. While the reliability of dispersion-corrected energy trends to reproduce solution phase or solid state conditions has been frequently debated,[49-52] direct comparison with experiment undertaken in condensed phase should be done with care. There is no doubt that having access to accurate experimental reference values in both gas ${ }^{[46}$ and crystalline phase ${ }^{[50]}$ would be beneficial to further investigate this matter. The proper description of binding energies in the excited-states also holds the promise of additional challenges, as current atompairwise dispersion corrections are not devised to capture the fundamental difference between ground- and excited- states.

\section{Analyzing Non-covalent Interactions}

Though extremely powerful and applicable to relatively large molecules, the quantum chemistry methods currently used (and used in the previous section) rely on sophisticated quantities such as the electronic density or the multidimensional wavefunction. On this basis, chemical insight is often lost in the complexity of the employed method, and the output data become more difficult to rationalize than data obtained through very simple approximations (e.g. Hückel theory). If one wishes to gain further insight into the origin of the phenomena governing non-covalent interactions, it is essential to not only know the total energy, electron density or geometry, but also the contributions from individual types of energy contributions (electrostatics, Pauli repulsion, dispersion, polarization, and charge transfer) or the relationships between chemical patterns and properties of interest. For this purpose, the computational community has been developing valuable tools ranging from rigorous energy decomposition schemes to simple qualitative models. Our laboratory has expended a great deal of effort to introduce original quantum chemical approaches that specifically enhance understanding of the nature of inter- and intramolecular interactions. ${ }^{[10-15]}$

For instance, we have broadened the field of applicability of energy decomposition schemes based on both the supermolecular[13] approach and perturbation theory. ${ }^{[10]}$ As stressed earlier, dispersion interactions are not only dominate in the binding of van der Waals complexes, but occur within all molecules. We have addressed a very difficult, yet pressing issue: the energy decomposition of non-covalent interactions occurring within molecules. ${ }^{[10]}$ Among the various existing intermolecular decomposition schemes, SymmetryAdapted Perturbation Theory (SAPT) ${ }^{[53]}$ may be considered amongst the most successful, but an equivalent energy decomposition scheme available for intramolecular interactions is lacking. ${ }^{[1]}$ The deriva- 


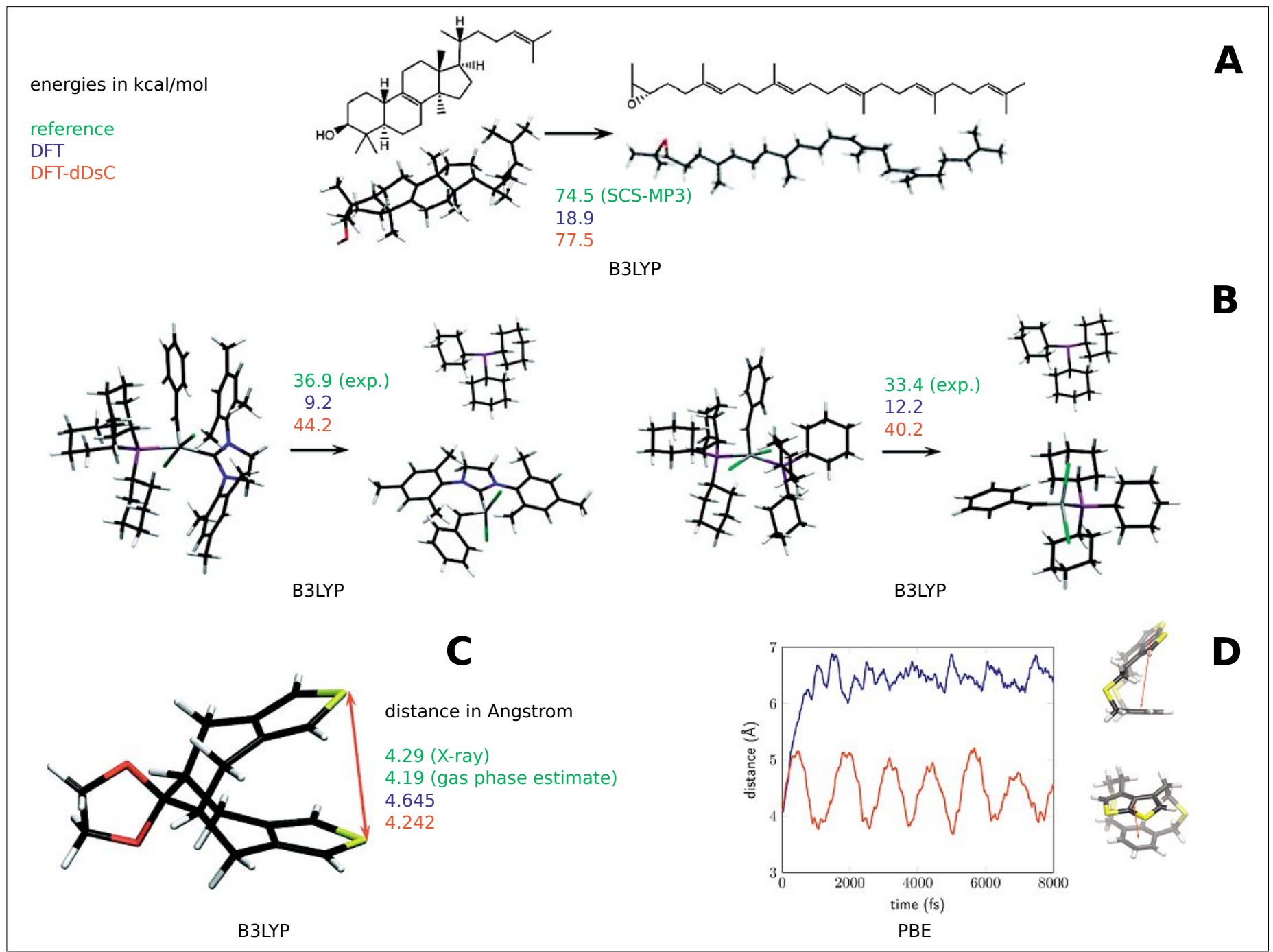

Fig. 1. Set of illustrative chemical phenomena ${ }^{[8,45]}$ poorly described by standard density functionals (e.g. B3LYP and PBE) and corrected by dDsC. The reference values for $\mathbf{A}$ are computed at the SCS-MP3/CBS level. For $\mathbf{B}$ and $\mathbf{C}$, the experimental values ${ }^{[46-48]}$ serve as a benchmark. The DFT energies for $\mathbf{A}$ and $\mathbf{B}$ are computed with the def2-TZVP basis set. The cc-pVTZ and 6-31G* basis sets were used in $\mathbf{C}$ and $\mathbf{D}$ respectively.

tion of an intramolecular variant of SAPT has been one of our long-term goals. As a preliminary step, we derived a zeroth-order wavefunction and energy ${ }^{[10]}$ that excludes the interactions between fragments of interest by making use of strictly localized orbitals and the chemical Hamiltonian approach. This formalism is suitable for the application of biorthogonal perturbation theory ${ }^{[54]}$ and for the derivation of the first and second order energy formulae, which will enable the decomposition of the intramolecular interaction energy into physically meaningful and intuitive energy quantities akin to intermolecular SAPT. The availability of such an approach would open the door to address classes of problems previously inaccessible.

Next to energy decomposition schemes, molecular scalar fields can be very insightful to analyze chemical bonding patterns of ground state molecules (e.g. ELF, ${ }^{[55,56]}$ LOL, $\left.{ }^{57]} \mathrm{SEDD}^{[58]}\right)$ or non-covalent interactions (e.g. NCI $\left.{ }^{[59]}\right)$. These fields provide direct information concerning the electronic distribution and character of chemical bonds, lone pairs, and about regions where molecules or intramolecular fragments interact non-covalently. Our LOLIPOP ${ }^{[12]}$ criterion (see upper right image of Scheme 1 ) is based on a function of the kinetic energy density called the Localized Orbital Locator (LOL ${ }^{[57]}$ ) as originally proposed by the group of Becke. LOLIPOP relies upon a realization of fundamental importance, namely that $\pi$-depleted polyaromatic molecules present superior $\pi$-stacking ability compared to rich $\pi$-electron cores. The practical utility of LOLIPOP was demonstrated by identifying tailored chemosensors that were experimentally shown to display remarkable sensitivity and selectivity towards caffeine. ${ }^{60]}$ More recently, our group has elaborated a new density-dependent scalar field, the Density Overlap Regions Indicator (DORI), ${ }^{[14]}$ which simultaneously detects both covalent bonding patterns and noncovalent interactions. DORI has been exploited to quantify electronic compactness in molecular aggregates relevant to organic electronics and to identify a correlation between enhanced charge carrier mobility and electronic compactness. ${ }^{[14,61]}$

Together, these approaches have already enhanced our fundamental comprehension of phenomena governed by intermolecular and intramolecular interactions. Our current effort is focused on applying newly acquired knowledge concerning non-covalent interactions to systems relevant to the field of organic electronics. In particular, we aim to identify unprecedented trends and structural motifs that lead to assemblies of $\pi$-conjugated molecules exhibiting improved semiconducting properties (bottom right image in Scheme 1). ${ }^{\text {62] }}$ In connection with the previous section, this specific objective is also connected with achieving predictive and accurate modeling of these large molecular architectures dominated by ubiquitous van der Waals interactions. ${ }^{[63]}$

\section{Conclusion}

An overview of some of the challenges associated with the description and analy- 
sis of 'weak' intra- and intermolecular interactions using quantum chemical approaches has been provided. The most widely used electronic structure framework does not properly describe these crucial interactions. On this basis, we summarized a handful of schemes developed in our laboratory to address the inability of standard density functional approximations to provide the correct $-\mathrm{C}_{6} / \mathrm{R}^{6}$ dependence, placing special emphasis on intramolecular interactions. Next to delivering robust atom pairwise dispersion corrections to DFT-based methods, we will continue to introduce electronic structure approaches for analyzing and possibly predicting phenomena governed by intra- and intermolecular interactions. Many challenges remain, including achieving accurate description beyond ground state phenomena and devising the next generation of DFT methods that can overcome several shortcomings of density functional approximations.

\section{Acknowledgements}

The author warmly thanks all the past and present talented and highly dedicated students and post-docs, who carried out the work. Most of the research summarized in this article is associated with the work of Stephan N. Steinmann, Jerome F. Gonthier, Eric Bremond, Piotr da Silva, Hongguang Liu, and Riccardo Petraglia. The financial support from the Sandoz family foundation, the Swiss NSF Grant no. 20021_137529, the European Research Council (ERC Grants 306528, 'COMPOREL'), and EPFL is greatly acknowledged.

Received: June 26, 2014

[1] C. Corminboeuf, Acc. Chem. Res. 2014, DOI 10.1021/ar400303a

[2] S. N. Steinmann, M. D. Wodrich, C. Corminboeuf, Theor. Chem. Acc. 2010, 127, 429.

[3] S. N. Steinmann, C. Piemontesi, A. Delachat, C. Corminboeuf, J. Chem. Theory Comput. 2012, $8,1629$.

[4] S. N. Steinmann, C. Corminboeuf, J. Chem. Theory Comput. 2012, 8, 4305.

[5] S. N. Steinmann, G. Csonka, C. Corminboeuf, J. Chem. Theory Comput. 2009, 5, 2950.
[6] S. N. Steinmann, C. Corminboeuf, J. Chem. Theory Comput. 2010, 6, 1990.

[7] S. N. Steinmann, C. Corminboeuf, J. Chem. Phys. 2011, 134, 044117.

[8] S. N. Steinmann, C. Corminboeuf, J. Chem. Theory Comput. 2011, 7, 3567.

[9] S. N. Steinmann, C. Corminboeuf, Chimia 2011, 65, 240.

[10] J. F. Gonthier, C. Corminboeuf, J. Chem. Phys. 2014, 140,154107 .

[11] J. F. Gonthier, C. Corminboeuf, Chimia 2014, $68,221$.

[12] J. F. Gonthier, S. N. Steinmann, L. Roch, A. Ruggi, N. Luisier, K. Severin, C. Corminboeuf, Chem. Commun. 2012, 48, 9239.

[13] S. N. Steinmann, C. Corminboeuf, W. Wu, Y. Mo, J. Phys. Chem. A 2011, 115, 5467.

[14] P. de Silva, C. Corminboeuf, J. Chem. Theory Comput. 2014, DOI: $10.1021 / \mathrm{ct} 500490 \mathrm{~b}$.

[15] J. F. Gonthier, M. D. Wodrich, S. N. Steinmann, C. Corminboeuf, Org. Lett. 2010, 12, 3070.

[16] W. Kohn, L. J. Sham, Phys. Rev. 1965, 140, A1133.

[17] A. D. Becke, J. Chem. Phys. 1993, 98, 5648

[18] P. J. Stephens, F. J. Devlin, C. F. Chabalowski, M. J. Frisch, J. Phys. Chem. 1994, 98, 11623.

[19] S. Kristyan, P. Pulay, Chem. Phys. Lett. 1994, $229,175$.

[20] J. M. Perez-Jorda, A. D. Becke, Chem. Phys. Lett. 1995, 233, 134.

[21] P. Hobza, J. Sponer, T. Reschel, J. Comput. Chem. 1995, 16, 1315.

[22] Y. Zhang, W. Pan, W. Yang, J. Chem. Phys. 1997, 107, 7921.

[23] J. Klimes, A. Michaelides, J. Chem. Phys. 2012, 137, 120901.

[24] K. Lee, E. D. Murray, L. Kong, B. I. Lundqvist, D. C. Langreth, Phys. Rev. B 2010, 82, 081101.

[25] O. A.Vydrov, T. Van Voorhis, J. Chem. Phys. 2010, 133, 244103.

[26] Y. Zhao, D. G. Truhlar, J. Phys. Chem. A 2004, $108,6908$.

[27] S. Grimme, J. Chem. Phys. 2006, 124, 034108.

[28] J. G. Angyan, I. C. Gerber, A. Savin, J. Toulouse, Phys. Rev. A 2005, 72, 012510.

[29] E. Goll, H. J. Werner, H. Stoll, Phys. Chem. Chem. Phys. 2005, 7, 3917.

[30] H. Eshuis, J. Bates, F. Furche, Theor. Chem. Acc. 2012, 131, 1084.

[31] O. A. von Lilienfeld, I. Tavernelli, U. Rothlisberger, D. Sebastiani, Phys. Rev. Lett. 2004, 93, 153004.

[32] I. D. Mackie, G. A. DiLabio, J. Phys. Chem. A 2008, 112, 10968.

[33] Y. Zhao, D. Truhlar, Theor. Chem. Acc. 2008, $120,215$.

[34] S. Grimme, WIREs Comput. Mol. Sci. 2011, 1, 211.

[35] S. Grimme, J. Comput. Chem. 2004, 25, 1463.

[36] S. Grimme, J. Comput. Chem. 2006, 27, 1787.
[37] S. Grimme, J. Anthony, S. Ehrlich, H. Krieg, $J$. Chem. Phys. 2010, 132, 154104.

[38] E. R. Johnson, A. D. Becke, J. Chem. Phys. 2005, 123,024101

[39] A. Tkatchenko, M. Scheffler, Phys. Rev. Lett. 2009, 102, 073005.

[40] T. Sato, H. Nakai, J. Chem. Phys. 2009, 131, 224104.

[41] A. Tkatchenko, J. DiStasio, A. Robert, R Car, M. Scheffler, Phys. Rev. Lett. 2012, 108 236402.

[42] A. Krishtal, C. Van Alsenoy, P. Geerlings, $J$. Chem. Phys. 2014, 140, 184105.

[43] R. Ahlrichs, R. Penco, G. Scoles, Chem. Phys. 1977, 19, 119.

[44] Q. Wu, W. Yang, J. Chem. Phys. 2002, 116, 515

[45] E. Bremond, N. Golubev, S. N. Steinmann, C. Corminboeuf, J. Chem. Phys. 2014, 140, 18 A516.

[46] S. Torker, D. Merki, P. Chen, J. Am. Chem. Soc. 2008, 130, 4808

[47] K. M. Knoblock, C. J. Silvestri, D. M. Collard, J. Am. Chem. Soc. 2006, 128, 13680.

[48] J. Moellmann, S. Grimme, Phys. Chem. Chem. Phys. 2010, 12, 8500.

[49] S. Schmidt, G. Abkai, T. Rosendahl, F Rominger, P. Hoffmann, Organometallics 2013, $32,1044$.

[50] J. Moellmann, S. Grimme, Organometallics 2013, 32, 3784.

[51] H. Jacobsen, L. Cavallo, ChemPhysChem 2012 13,562 .

[52] S. Grimme, ChemPhysChem 2012, 13, 1407.

[53] B. Jeziorski, R. Moszynski, K. Szalewicz, Chem. Rev. 1994, 94, 1887.

[54] I. Mayer, Int. J. Quant. Chem. 1983, 23, 341.

[55] A. D. Becke, K. E. Edgecombe, J. Chem. Phys. 1990, 92, 5397.

[56] B. Silvi, A. Savin, Nature 1994, 371, 683

[57] H. Schmider, A. Becke, J. Mol. Struct.: THEOCHEM 2000, 527, 51 .

[58] P. De Silva, J. Korchowiec, T. A. Wesolowski, ChemPhysChem 2012, 13, 3462.

[59] E. R. Johnson, S. Keinan, P. Mori-Sanchez, J. Contreras-Garcia, A. J. Cohen, W. Yang, J. Am. Chem. Soc. 2010, 132, 6498.

[60] N. Luisier, A. Ruggi, S.N. Steinmann, L. Favre, N. Gaeng, C. Corminboeuf, K. Severin, Org. Bio. Chem. 2012, 10, 7487.

[61] J. Gebers, L. Hartmann, M. Schaer, S. Suarez, P. Bugnon, H.-G. Steinrueck, A. Magerl, M. Brinkmann, R. Petraglia, C. Corminboeuf, $\mathrm{H}$. Frauenrath, submitted, 2014.

[62] H. Liu, E. Bremond, A. Prlj, J. F. Gonthier, C. Corminboeuf, J. Phys. Chem. Lett. 2014, 5 , 2320.

[63] R. Marty, R. Szilluweit, A. Sanchez-Ferrer, S. Bolisetty, J. Adamcik, R. Mezzenga, E.C. Spitzner, M. Feifer, S. N. Steinmann, C. Corminboeuf, H. Frauenrath, ACS Nano 2013, 7,8498 . 